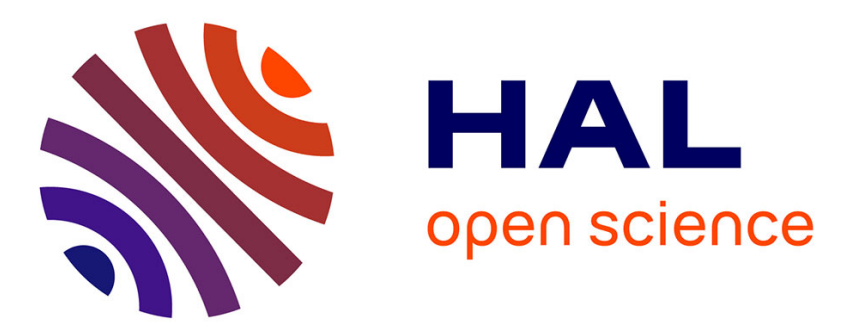

\title{
Basic Mechanical Properties of Wet Granular Materials: A DEM Study
}

Vinh Du Than, Saeed Khamseh, Anh Minh A.M. Tang, Jean-Michel Pereira, François Chevoir, Jean-Noël Roux

\section{- To cite this version:}

Vinh Du Than, Saeed Khamseh, Anh Minh A.M. Tang, Jean-Michel Pereira, François Chevoir, et al.. Basic Mechanical Properties of Wet Granular Materials: A DEM Study. Journal of Engineering Mechanics - ASCE, 2017, 143 (1), 10.1061/(ASCE)EM.1943-7889.0001043 . hal-01515873

\section{HAL Id: hal-01515873 \\ https://hal-enpc.archives-ouvertes.fr/hal-01515873}

Submitted on 3 May 2017

HAL is a multi-disciplinary open access archive for the deposit and dissemination of scientific research documents, whether they are published or not. The documents may come from teaching and research institutions in France or abroad, or from public or private research centers.
L'archive ouverte pluridisciplinaire HAL, est destinée au dépôt et à la diffusion de documents scientifiques de niveau recherche, publiés ou non, émanant des établissements d'enseignement et de recherche français ou étrangers, des laboratoires publics ou privés. 


\title{
BASIC MECHANICAL PROPERTIES
}

\section{OF WET GRANULAR MATERIALS: A DEM STUDY}

\author{
Vinh Du Than ${ }^{1}$, Saeed Khamseh ${ }^{1}$,

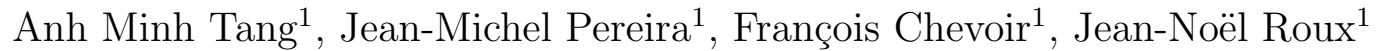

\section{ABSTRACT}

We use discrete, grain-level numerical simulations of a model granular assembly, made of spherical balls, to investigate the influence of a small amount of an interstitial wetting liquid, forming capillary bridges between adjacent particles, on two basic aspects of granular material rheology: (i) the plastic response in isotropic compression, and (ii) the critical state under monotonic shear strain, and its generalization to steady, inertial flow. Tensile strength $F_{0}=\pi \Gamma a$, in contacts between beads of diameter $a$ joined by a small meniscus of a liquid with surface tension $\Gamma$, introduces a new force scale and a new dimensionless control parameter, $P^{*}=a^{2} P / F_{0}$, for grains of diameter $a$ under confining stress $P$. Under low $P^{*}$, as cohesion dominates, capillary cohesion may stabilize very loose structures. Upon increasing pressure $P$ in isotropic compression, such structures gradually collapse. The resulting irreversible compaction is well described by the classical linear relation between $\log P^{*}$ and void ratio in some range, until a dense structure forms which retains its stability without cohesion as confinement dominates for large $P^{*}$. In steady shear flow, with uniform velocity gradient $\dot{\gamma}=\frac{\partial v_{1}}{\partial x_{2}}$ under normal stress $P=\sigma_{22}$, the apparent internal friction coefficient, which we define as $\mu^{*}=\frac{\sigma_{12}}{\sigma_{22}}$, depends on $P^{*}$ and inertial number (reduced shear rate) $I=\dot{\gamma} \sqrt{\frac{m}{a P}}$, and so does solid fraction $\Phi$. The material exhibits, as $P^{*}$ decreases, a strongly enhanced resistance to shear (larger $\mu^{*}$ ). In the quasistatic limit, for $I \rightarrow 0$, it is roughly predicted by a simple effective pressure assumption, by which the capillary forces are deemed equivalent to

\footnotetext{
${ }^{1}$ Université Paris Est, Laboratoire Navier, 2 Allée Kepler, Cité Descartes, 77420 Champs-sur-Marne, France
}

1 Than et al., revised version, July 9, 2015 
an isotropic pressure increase applied to the dry material, as long as $P^{*} \geq 1$, while the yield criterion approximately assumes the Mohr-Coulomb form. At lower $P^{*}$, such models tend to break down as liquid bonding, causing connected clusters to survive over significant strain intervals, strongly influences the microstructure. Systematic shear banding is observed at very small $P^{*}$.

Keywords: granular materials, cohesion, capillary forces, effective pressure, Mohr-Coulomb, DEM

\section{INTRODUCTION}

Over the last decades our understanding of the microstructural and micromechanical origins of macroscopic granular material rheology have greatly benefitted from the development of numerical simulation methods of the so-called discrete element type (DEM), in which the motions and interactions of individual grains are modeled (Radjaï and Dubois 2011). In particular, two basic concepts, which had previously been identified and exploited in process, chemical and geomechanical engineering, were revisited, and supported by micromechanical interpretations. One is the random close packing (RCP) state (Cumberland and Crawford 1987), the configuration of a granular assembly maximizing density under the constraint of mutual non-interpenetrability of the grains, without any specific ordering. The $\mathrm{RCP}$ state is a stable equilibrium state of an isotropically compressed assembly of rigid, frictionless grains (Agnolin and Roux 2007a; Donev et al. 2005; O'Hern et al. 2003), and its characteristics, most notably its solid fraction, are remarkably reproducible, irrespective of the dynamical assembling method. Furthermore, because frictionless particle assemblies appear to be devoid of dilatancy - which has been explicitly checked for disks and spherical beads (Peyneau and Roux 2008a), and for polygonal shapes in 2D (Azéma et al. 2015) - the same solid fraction (about 0.64 for identical beads) is obtained on preparing, without any friction mobilization, packings under different, possibly anisotropic, conditions (Silbert et al. 2002; Peyneau and Roux 2008b). In the presence of friction, many different states can be observed, varying in density and coordination number (Agnolin and Roux 2007a; Magnanimo 
et al. 2008), even if stresses and microstructure are isotropic. The second traditional notion which has been revisited by DEM, with due attention to its microscopic foundations, is that of the critical state, in the sense of soil mechanics (Wood 1990; Mitchell and Soga 2005): the steady state of plastic flow attained, irrespective of the initial state, after large enough strain in monotonically, quasistatically sheared material. The critical state has been shown (Radjaï et al. 2004; Rothenburg and Kruyt 2004; Radjaï et al. 2012; Kruyt and Rothenburg 2014) to be an attractor state for all variables characterizing internal structure, micromorphology and forces, such as coordination numbers, fabric tensors or friction mobilization, as well as for stresses and solid fraction. Upon increasing the shear rate, the material behavior is affected by inertial effects, and the internal state of the homogeneously sheared material depends, under controlled normal stress $P$, on inertial number $I$ (as defined in the abstract), the quasi-static critical state corresponding to the limit of $I \rightarrow 0$. This generalization of the critical state to $I$-dependent steady homogeneous shear flows has led to the formulation of efficient constitutive laws for dense granular flows (Forterre and Pouliquen 2008; Andreotti et al. 2013), in terms of the $I$ dependence of internal friction coefficient $\mu^{*}$ and solid fraction $\Phi$. The RCP state (or another well-controlled homogeneous isotropic packing state) on the one hand, and the critical state, on the other hand, correspond to the initial and the final states in many relevant mechanical tests - typically one starts from some isotropic packing, of which the RCP is an important limiting case, and one imposes a loading path leading to the critical state (Thornton 2000; Radjaï et al. 2004). Their interest also stems from their lack of dependence on many features and parameters governing contact behavior, especially dynamical ones, but also elastic contact stiffnesses, in the frequent case of negligible contact deflections (Roux and Chevoir 2011). On introducing new models for grains, with such features as rolling resistance or angularity (Azéma et al. 2013; Saint-Cyr et al. 2012; Estrada et al. 2011), it is natural to first investigate microstructural and mechanical properties of RCP and critical states. Cohesive forces in contacts significantly affect both isotropic packings (Gilabert et al. 2007) and steady shear flows (Rognon et al. 2006). 
The present paper states some essential results obtained by DEM simulations, for both isotropically compressed static assemblies, and $I$-dependent steady uniform shear flows, with special emphasis on the critical state in the limit of $I \rightarrow 0$, in the case of a model of wet spherical grains. Compared to similar numerical studies in the literature (Richefeu et al. 2006; Scholtès et al. 2009b) the ones presented here investigate looser structures, which could not be observed with dry grains - as evidenced in experiments with sands (Bruchon et al. 2013a; Bruchon et al. 2013b). While both situations should be more extensively studied, in more detailed publications (Khamseh et al. 2015; Than et al. 2015), in which thorougher investigations of microscopic aspects will be presented, some salient features of isotropic compression and steady shear flows are described, stressing the differences with dry, cohesionless materials.

The paper is organized in the following way. Once the model material and the interactions are suitably described in the forthcoming section ("Model material and simulation ingredients"), the two main parts of the paper separately address these two important aspects of wet granular material rheology: "Isotropic assembly and compression", and then "Dense shear flow and critical states". The final Conclusion section sums up the results and puts them in perspective.

\section{MODEL MATERIAL AND SIMULATION INGREDIENTS}

\section{Stress and Strain Control}

Our model material is an assembly of $N$ equal-sized spherical beads of diameter $a$. The simulation cell is a rectangular cuboid, with edges, of lengths $\left(L_{\alpha}\right)_{1 \leq \alpha<3}$, parallel to coordinate axes, periodic in all three directions. We control all three diagonal stress components in isotropic compression, and wait for equilibrium conditions, as in Agnolin and Roux (2007a), to be satisfied within a preset tolerance. In shear tests a granular flow is imposed in direction 1 , with a gradient in direction $x_{2}$, defining shear rate $\dot{\gamma}=\frac{\partial v_{1}}{\partial x_{2}}$. In that case, the periodicity in direction 2 is implemented through the Lees-Edwards procedure (Allen and Tildesley 
1987), length $L_{2}$ is allowed to vary in response to the enforced condition of constant normal stress $\sigma_{22}$, while lengths $L_{1}$ and $L_{3}$ are kept fixed - as in Peyneau and Roux (2008a).

\section{Force Model: Elasticity and Friction}

The spherical beads are assumed to be made of a material with Young modulus E and Poisson ratio $\nu$. Coulomb friction applies in the contacts, with friction coefficient $\mu$. Elastic-frictional contact forces are modeled with a simplified Hertz-Mindlin-Deresiewicz force law (Agnolin and Roux 2007a). The normal Hertz force $F_{N}$ depends on contact deflection $h$ as

$$
F_{N}=\frac{\tilde{E} \sqrt{a}}{3} h^{3 / 2}
$$

in which we introduced notation $\tilde{E}=E /\left(1-\nu^{2}\right)$. The adopted simplified form of tangential elasticity (Agnolin and Roux 2007a) involves a constant ratio $(2-2 \nu) /(2-\nu)$ of tangential $\left(K_{T}\right)$ to normal $\left(K_{N}\right)$ stiffnesses in contacts. Both depend on $F_{N}$, as, from $(1), K_{N}=\frac{d F_{N}}{d h} \propto$ $F^{1 / 3}$. With such laws one should avoid spurious creation of elastic energy, and therefore $K_{T}$ is suitably rescaled in cases of decreasing normal force (Elata and Berryman 1996). Details on the elastic model, on the enforcement of the Coulomb condition,

$$
\left\|\mathbf{F}_{T}\right\| \leq \mu F_{N}
$$

and on the objective implementation of the force law, with due account of all possible motions of a pair of contacting grains, are given by Agnolin and Roux (2007a). Our simulations are carried out with the elastic properties of glass beads $(\tilde{E}=77 \mathrm{GPa})$ and the intergranular friction coefficient, $\mu$, is kept equal to 0.3 in the present study.

Estimating the typical contact deflection under confining stress $P$ leads to the definition of a dimensionless stiffness parameter $\kappa$ (Radjaï and Dubois 2011), such that $h / a \propto \kappa^{-1}$. For a Hertzian contact, one may use (Agnolin and Roux 2007a)

$$
\kappa=\left(\frac{\tilde{E}}{P}\right)^{2 / 3}
$$


In our shear test simulations we keep $\kappa=8400$, corresponding to glass beads under pressure $100 \mathrm{kPa}$. It is deemed large enough to approach the limit of rigid grains with good accuracy.

Viscous damping terms oppose normal relative motion of contacting grains, and are chosen to correspond to a restitution coefficient close to zero in normal collisions. Such terms were shown (da Cruz et al. 2005; Peyneau and Roux 2008a) to have negligible influence in the slow compression steps and shear flows of the present study.

\section{Force Model : Capillary Attraction}

An interstitial wetting liquid, introduced in small amounts, preferentially localizes at contacts or between close neighboring grains, forming liquid bridges transmitting capillary forces. Such a liquid bridge, or meniscus, is sketched in Fig. 1. We consider a perfectly wetting liquid, with contact angle $\theta$ equal to zero. In accordance with some observations (Herminghaus 2005), we assume that the menisci only form as particles come into contact. If contacting grains move apart from one another, and are separated by distance $h$, the liquid bridge remains stable, transmitting an $h$-dependent force, until the gap, $h$, reaches a certain rupture distance $D_{0}$, as observed in (Kohonen et al. 2004). $D_{0}$ relates to meniscus volume $V$ as $D_{0} \simeq V^{1 / 3}$ (Lian et al. 1993; Willett et al. 2000; Pitois et al. 2000).

The attractive force between particles separated by distance $h \leq D_{0}$ is modeled within the Maugis approximation (Maugis 1987), appropriate for small enough meniscus volume. The maximum attractive force (tensile strength) is reached for contacting particles, and equal, according to this model, to $F_{0}=\pi a \Gamma$ ( $\Gamma$ is the liquid surface tension) independent of the meniscus volume. The capillary force varies with gap $h$ between particle surfaces as

$$
F^{\text {Cap }}= \begin{cases}-F_{0} & h \leq 0 \\ -F_{0}\left[1-\frac{1}{\sqrt{1+\frac{2 V}{\pi a h^{2}}}}\right] & 0<h \leq D_{0} \\ 0 & h>D_{0}\end{cases}
$$

(One should note that $h<0$ corresponds to an elastic deflection of the particles in contact, 
and keep in mind that a nonvanishing distant force, $F^{\mathrm{Cap}}(h)$ with $h>0$, is only possible if the grains have been in contact and did not move apart farther than distance $D_{0}$ ever since). This formula is a simpler, analytical form of the toroidal approximation with the "gorge method" (Lian et al. 1993) for the capillary force in a meniscus. Alternative forms of the attractive force law (Willett et al. 2000; Soulié et al. 2006; Radjaï and Richefeu 2009) might actually be more accurate. We found (Khamseh et al. 2015) that the macroscopic results were not affected upon changing the force law, were very moderately influenced by saturation within the pendular range, but did significantly change upon suppressing the capillary hysteresis (i.e., assuming menisci to form as soon as a pair of grains approach within rupture distance $D_{0}$ ). It is important to recall that the Coulomb inequality, as written in (2), applies to the elastic component of the normal force only, to which the negative (attractive) capillary term should be added. Thus, in an isolated grain pair bonded by a meniscus, at equilibrium the repulsive elastic force is equal to $F_{0}$, and the contact may transmit a tangential force at most as large as $\mu F_{0}$.

The morphology of partially saturated granular materials depends on the liquid content (Mitarai and Nori 2006; Kudrolli 2008). The present study, like a number of previous ones (Richefeu et al. 2006; Radjaï and Richefeu 2009; Scholtès et al. 2009a), is restricted to the pendular state of low saturations, in which the wetting liquid is confined in bonds or menisci joining contacting grains. Liquid saturation $S$ is defined as the ratio of liquid volume $\Omega_{l}$ to interstitial volume $\Omega_{v}$, the total system volume being denoted as $\Omega$. Writing $\Omega_{g}$ for the volume of all $N$ solid grains in the system (such that $\Omega=\Omega_{g}+\Omega_{v}, S$ is related to meniscus volume $V$, solid fraction $\Phi=\Omega_{g} / \Omega=1-\Omega_{v} / \Omega=\frac{N \pi a^{3}}{6 \Omega}$ and wet coordination number $z$ (the average number of liquid bonds on one grain). As the liquid volume is $\Omega_{l}=\frac{z N V}{2}$, one has

$$
S=\frac{\Omega_{l}}{\Omega_{v}}=\frac{z N V}{2(1-\Phi) \Omega}=\frac{3 z}{\pi} \frac{\Phi}{1-\Phi} \frac{V}{a^{3}} .
$$

In our study, we fix the value of meniscus volume $V$, equal to $10^{-3} a^{3}$ in all results presented 
in this paper. Such a choice does not conserve the total liquid volume (proportional to the varying coordination number $z$ of liquid bonds) - but this is, as we could check (Khamseh et al. 2015), quite an innocuous drawback.

The pendular state to which our model applies is relevant in some low (but not too small) saturation range. The upper saturation limit for the pendular state corresponds to the merging of the menisci pertaining to the same grain, which, considering a triangle of spherical grains in mutual contact, happens as soon as the filling angle $\varphi$ (see Fig. 1 ) reaches $\pi / 6$. The analytical formula for $V$ (Lian et al. 1993), within the toroidal approximation, as a function of $\varphi$ (setting $h=0$, and $\theta=0$ ), then yields $\frac{V}{a^{3}} \simeq 8 \cdot 10^{-3}$. Eq. (5) then predicts a maximum saturation between 0.05 and 0.1, similar to experimental observations (Herminghaus 2005; Mitarai and Nori 2006). On the other hand, the minimum saturation $S_{\min }$ for bridges to form and join neighbouring grains might be roughly estimated upon introducing a roughness scale $\delta$, assuming a liquid layer of thickness $\delta$ coats the surface of the grains, as

$$
S_{\min }=\frac{6 \Phi \delta}{(1-\Phi) a} .
$$

For $\Phi=0.5$ and $\delta \sim 10^{-4} a, S_{\min }$ is of the order of $10^{-3}$, as observed in experiments (Herminghaus 2005). Using (5), and typical values of $z$ and $\Phi$, this sets a lower bound to meniscus volume, of order $10^{-4} a^{3}$.

Capillary attraction introduces force scale $F_{0}$ in the model, whence the definition of a reduced pressure, comparing applied stress $P$ (isotropic pressure in compression, or controlled normal stress $\sigma_{22}=P$ for shear flows) to contact tensile strength $F_{0}$, as

$$
P^{*}=\frac{a^{2} P}{F_{0}}=\frac{a P}{\pi \Gamma} .
$$

As observed with different cohesive granular models, mostly in two dimensions (2D) (Gilabert et al. 2007), we expect strong effects of cohesive forces, possibly very loose equilibrium microstructures for $P^{*} \ll 1$, while the properties of cohesionless systems are retrieved in the 
limit of large $P^{*}$. Our simulations are carried out with spheres of diameter $a=0.115 \mathrm{~mm}$,

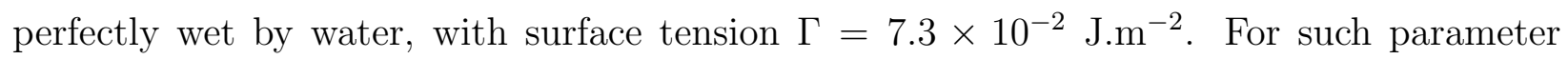
values, one has $P^{*}=1$ for a pressure $P$ equal to $2 \mathrm{kPa}$. While this is admittedly a rather low pressure for most geotechnical applications, it might be relevant in other fields (e.g., in some chemical engineering process), and the results are also, beyond wet grain models, more generally indicative of the influence of attractive forces of small range in granular assemblies. Another important issue is the possible influence of the initial microstructure assembled under low $P^{*}$ on the material properties under larger confining stresses. In the following most results are expressed in terms of dimensionless control parameters.

\section{ISOTROPIC ASSEMBLY AND COMPRESSION}

We studied the important irreversible configuration changes entailed by a pressure cycle starting with a low value of $P^{*}$, of order $10^{-2}$ or $10^{-3}$, with an initial state that cannot be observed without cohesive forces. Maximum pressures are such that $P^{*} \gg 1$. Our parameter choice is such that $\kappa=114000$ for $P^{*}=1$ and $\kappa=5300$ for $P^{*}=100$, which is still high enough a value for contact deflections to be irrelevant (Roux and Chevoir 2011). Consequently, our results, if expressed as dimensionless quantities functions of $P^{*}(\kappa$ being large enough to be irrelevant), may apply to systems of wet spherical grains with arbitrary diameter, liquid surface tension and wetting angle.

\section{Loose initial states}

Previous 2D simulations of cohesive systems by Gilabert et al. (2007) made it clear that cohesive forces play an important part in the assembling stage. It is thus necessary to assemble wet grains, rather than introduce liquid bridges into previously assembled dry grain configurations. As in the 2D studies, we found it possible to assemble low density initial configurations by the following procedure. First, disordered assemblies of grains (comprising 4000 particles) with solid fraction $\Phi_{0}$ are prepared, using either random insertion or crystal melting with event-driven, energy-preserving dynamics (Agnolin and Roux 2007a). Then, particles are attributed random velocities, drawn according to a Gaussian distribution with 
zero mean and variance $V_{0}^{2}$, and left to interact with the force laws introduced in the previous section, within a periodic simulation cell of fixed size and shape. Capillary attraction induces the formation of clusters of grains joined by liquid bridges, and this step of the calculation is stopped when all particles belong to one single such cluster, and the configuration is regarded as sufficiently equilibrated. (Tolerances on equilibrium requirements are expressed in terms of typical contact force $F_{1}$ as, $10^{-4} F_{1}$ for force balance, or $10^{-4} a F_{1}$ for torque balance - with $F_{1}=F_{0}$ in the assembling stage). The structure of such initial configurations depends on imposed solid fraction $\Phi_{0}$, which we chose equal to 0.3 in the main simulation series. It also depends on velocity $V_{0}$. The latter should be compared to the characteristic velocity $V^{*}=\sqrt{\frac{D_{0} F_{0}}{m}}$, which is proportional to the relative velocity that is necessary to separate a pair of grains, overcoming the potential energy of capillary force (4). Here, choosing $V_{0} / V^{*}=0.2$, we could observe that the results corresponded to a low initial agitation limit. An important variable characterizing equilibrium configurations, the contact coordination number, $z_{c}$, is then barely larger than 4 - the isostatic (barely rigid) value (Agnolin and Roux 2007a). The coordination number of distant interactions, i.e., the average number of non-contacting neighbors connected to one grain through a capillary meniscus, which we denote as $z_{d}$, is equal to zero. This latter observation is explained by the capillary hysteresis: liquid bonds without contact only exist in pairs that have been in contact in the past. Thus, for low enough initial agitation velocity $V_{0}$, contacts, once formed in the assembling stage, do not break in the constant density aggregation stage.

\section{Compression curves}

\section{Loading procedure and measurements}

To study the compression of initially loose configurations, stabilized at $\Phi_{0}=0.3$ thanks to adhesive forces, a loading program is applied in which the isotropic pressure, $P$, is stepwise incremented, from a low value (corresponding to $P^{*}=10^{-3}$ ) up to $P^{*}=10000$. Steps are uniform on a logarithmic scale (i.e., pressure is multiplied by $10^{1 / 4}$ at each step). For each new value of applied pressure $\mathrm{P}$, one waits for equilibrium to be approached with good 
accuracy, and records the new configuration. To appreciate the irreversibility of the observed evolution, the loading program is a compression cycle with a decompression branch, on which the previously applied pressure levels are retraced back, down to the initial small value. This paper being only a brief account of salient behaviours in compression and in quasistatic shear flow, we do not present here a complete study of all properties and internal states of $P^{*}$ dependent isotropic configurations. We mainly focus on solid fraction $\Phi$, or, according to the soil mechanics presentation, on void ratio $e=-1+1 / \Phi$, and on contact $\left(z_{c}\right)$ and distant $\left(z_{d}\right)$ coordination numbers. A more complete parametric study (dependence on velocity $V_{0}$, meniscus volume, or various aspects of interaction laws such as capillary hysteresis and possible rolling friction) is also postponed to a forthcoming, more detailed publication (Than et al. 2015).

\section{Irreversible compression}

Fig. 2(a) shows the evolution of the void ratio in the isotropic compression of a system of 4000 beads, with the characteristics as described in the previous sections. The loading curve is composed of three parts: first (regime I), in range $P^{*} \leq 0.01$, the initial structure supports the pressure increase, and void ratio $e$ hardly departs from its initial value (equal to 2.33). Then, in a second stage (regime II), extending up to $P^{*} \simeq 10$, the system undergoes a fast compression, which becomes considerably slower at high pressures (regime III). On reducing $P^{*}$, only the density change occurring within regime III is reversed. As apparent on the second graph, Fig. 2(b), in which the reference wet system is compared to a dry (cohesionless) assembly of identical grains, regime III is parallel to the compression curve of dry grains, in which the small compression is due to contact elasticity, and, as shown by Agnolin and Roux (2007b), nearly reversible (in terms of density at least). Thus regime III marks the end of the plastic collapse of the loose structure stabilized by capillary forces.

The plastic compression behavior of the wet material is closely similar to the $2 \mathrm{D}$ results of Gilabert et al. (2008), and the void ratio curve in regime II might be represented with a linear variation with $\log P^{*}$, assuming $e_{\text {ref }}$ is the void ratio for some reference reduced 
pressure, $P_{\text {ref }}^{*}$ :

$$
e-e_{\mathrm{ref}}=-\lambda \log \frac{P^{*}}{P_{\mathrm{ref}}^{*}} .
$$

Coefficient $\lambda \simeq 0.36$ successfully describes the curve in interval $0.04 \leq P^{*} \leq 2$. Eq. 8 is classically used in soil mechanics for cohesive systems (Mitchell and Soga 2005). Upon unloading and reloading at various pressure levels along the compression curve, it is observed (as shown in Fig. 3) that a plastic response (irreversible structural rearrangement with density change) under isotropic pressure will be observed only if the maximum pressure the system has been subjected to in the past (the "overconsolidation pressure" of soil mechanics) is exceeded. This maximum pressure value appears to fully characterize the history dependence of the system in isotropic compression.

\section{Coordination numbers}

In the compression cycle, the coordination numbers of contact $\left(z_{c}\right)$ and distant $\left(z_{d}\right)$ interactions are shown in Fig. 4. Compared to density changes, those of the contact coordination number are remarkably small, as it increases from nearly 4 to about 4.8 after the full pressure cycle. As to the coordination number of menisci between distant grains, it starts at zero, due to the absence of contact opening in the assembling stage with small agitation velocity. Its increase to 2 in the course of irreversible compression signals the failure of the contact structure: the network gets rearranged as old contacts break, and are replaced by menisci connecting receding grain pairs, and new contacts form. The small change in $z_{c}$ is the net effect of contact creations and destructions. The final increase of $z_{c}$, accompanied by a decrease of $z_{d}$, in regime III (high pressures) is caused by the elastic compression, closing the gaps between non-contacting pairs, as in dry systems (Agnolin and Roux 2007b). We could check that the coordination numbers remain very nearly constant along the reversible paths of Fig. 3, which corresponds to unperturbed contact and meniscus networks.

Because of the capillary hysteresis of meniscus formation and breakage, only a proportion of neighbour pairs within rupture distance $D_{0}$ (defined in the presentation of the force model, 
in connection with Eq. 4), are connected by a liquid bridge. This proportion is initially zero, it increases with $P^{*}$, peaking at $70 \%$ in regime II, decreasing to about $55 \%$ in the denser systems (similar to the value $\sim 50 \%$ reported in the experiments by Kohonen et al. (2004)). Effect of drying or of saturating

In practice, one may act on a wet system by changing its saturation. The most drastic change should be obtained on entirely suppressing the capillary cohesion, either by drying, or by completely saturating the intergranular voids by the liquid. In numerical simulations, one may simply remove all capillary forces, leaving only the interactions present in a dry system. It is interesting to observe the effects of such an ideal transformation, carried out at various points along the irreversible compression curve. Fig. 5 shows the resulting void ratio evolution, if the system is deprived of capillary forces immediately before unloading at different pressure levels. This ideal drying or saturation step produces a sudden collapse (a brutal compression step), unless all irreversible compression has already taken place (as for points F and G on Fig. 5). (More gradual collapse due to progressive imbibition is reported in some experiments (Bruchon et al. 2013b)). In such a case, one may remove all capillary forces, as their mechanical role, at high $P^{*}$, is negligible. Remarkably, the final state after decompression keeps the same density, whether or not the system has been deprived of capillary cohesion.

\section{DENSE SHEAR FLOW AND CRITICAL STATE}

\section{Model Parameters}

The results reported here pertain to the same reference systems studied in isotropic compression, with $N=4000$, friction coefficient $\mu=0.3$ in the contacts, meniscus volume $V=10^{-3} a^{3}$. While stiffness number $\kappa$ is fixed, reduced pressure takes values $P^{*}=\infty$ (i.e., the dry case), 10, 5, 2, 1, 0.436 and 0.1 . The investigated range of $I$ values (varying from $10^{-4}$ to 0.562 by factors of $\sqrt{10}$ ) enables an accurate determination of the quasistatic limit, as well as an assessment of inertial effects. 
We focus on situations in which a uniform steady state might be identified under constant macroscopic shear rate $\dot{\gamma}$, after a transient stage (of a few unit strains at most). This turns out to exclude small values of $P^{*}$ : we could record homogeneous state parameters for $P^{*}=0.436$, but only partial information was gathered on the material state under $P^{*}=0.1$, since such systems flow inhomogeneously, localizing the velocity gradient within a narrow shear band (save for a restricted range of $I$ values of order 0.01 ).

\section{Constitutive laws}

Restricting their measurement to the higher values of $P^{*}$, we measure the (apparent) macroscopic friction coefficient $\mu^{*}=\frac{\sigma_{12}}{\sigma_{22}}$, and solid fraction $\Phi$, in steady homogeneous shear flows, with the results shown in Fig. 6. As in some published results (Rognon et al. 2006; Rognon et al. 2008), obtained in 2D with a model of cohesive disks, lower $P^{*}$ values increase $\mu^{*}$ and decrease the density of the sheared material. One may note, though, that the effect on $\mu^{*}$ is considerably larger in the 3D assembly of wet particles: even for $P^{*}=1$, when the attractive forces and the confining ones are of similar magnitude, the quasistatic internal friction coefficient, compared to its dry value $\left(P^{*}=\infty\right), \mu_{0}^{\infty}=0.335$, nearly doubles, at about 0.61 . It reaches $0.867 \pm 0.003$ at $P^{*}=0.436$. In addition to the values shown in the figure, limited data are available for $P^{*}=0.1$, in a range of $I$ (of order 0.01) for which shear banding does not occur. Such $I$ values are close enough, judging from the $I$ dependence of $\mu^{*}$ at different $P^{*}$ levels, to the quasistatic limit of $I \rightarrow 0$. We could then measure $\mu^{*} \simeq 1.62$ and $\Phi \simeq 0.435$.

As suggested by Rognon et al. (2006), for each $P^{*}$, a power law fit can describe the $I$ dependence of $\mu^{*}$ and $\Phi$, as in a number of studies of dry granular flows (Hatano 2007; Peyneau and Roux 2008a):

$$
\mu^{*}=\mu_{0}^{*}+C I^{\alpha}
$$

The data are compatible with a $P^{*}$-independent value of exponent $\alpha, \alpha \simeq 0.8$, while coefficient $C$ decreases for smaller $P^{*}$. In comparison with $\mu^{*}$, the solid fraction changes relatively 
little as a function of both $P^{*}$ and $I$ in the investigated range. Both quantities tend to depart slower from their quasistatic limit for $I \rightarrow 0$ as cohesive effects get stronger (for smaller $P^{*}$ ).

Void ratios $e_{0}=-1+1 / \Phi_{0}$ in the $P^{*}$-dependent critical states are compared to the values $e_{\text {iso }}\left(P^{*}\right)$ obtained in direct isotropic compression (normally consolidated states) in Fig. 7. The difference $e_{\text {iso }}-e_{0}$ is a decreasing function of $P^{*}$, but remains positive, and critical states are denser than isotropically compressed ones (this also applies to dry grains, $P^{*}=\infty$ - see the inserted subplot of Fig. 7). All normally consolidated isotropic states should therefore be regarded as loose: they have to contract under shear before approaching the critical state.

\section{Internal States, Microscopic Aspects}

Coordination numbers

Coordination numbers are shown in Fig. 8, as functions of $P^{*}$ for different $I$, showing a quasistatic limit to be closely approached at small $I$. A comparison to isotropic states obtained in compression (Fig. 4) reveals, unlike for the density, quite similar values of both $z_{c}$ and $z_{d}$ at given $P^{*}$. The number of contacts does not change much with $P^{*}$, while the number of distant interactions tends to increase with $P^{*}$. Faster flows (larger $I$ values) tend to break contacts, which results in smaller $z_{c}$ values, an effect partly compensated by the increase of $z_{d}$ : menisci survive contact openings with separation distances below $D_{0}$.

\section{Contributions to stresses}

Throughout the studied parameter range, stresses in the flow are dominated by force contributions:

$$
\sigma_{\alpha \beta}=\frac{1}{\Omega} \sum_{i<j} F_{i j}^{\alpha} r_{i j}^{\beta},
$$

the sum running over all pairs $i, j$ of grains interacting by force $F_{i j}, r_{i j}$ pointing from the center of $i$ to the center of $j$. This suggests a decomposition into contributions of different interactions. One may, e.g., isolate the contribution of distant interactions $\left(\sigma_{\alpha \beta}^{\mathrm{d}}\right)$ and contact interactions $\left(\sigma_{\alpha \beta}^{\mathrm{c}}\right)$, the latter being split into the contributions of tangential forces $\left(\sigma_{\alpha \beta}^{\mathrm{T}}\right)$ and 
normal ones $\left(\sigma_{\alpha \beta}^{\mathrm{N}, \mathrm{c}}\right)$ :

$$
\sigma_{\alpha \beta}=\sigma_{\alpha \beta}^{\mathrm{c}}+\sigma_{\alpha \beta}^{\mathrm{d}}=\sigma_{\alpha \beta}^{\mathrm{N}, \mathrm{c}}+\sigma_{\alpha \beta}^{\mathrm{T}}+\sigma_{\alpha \beta}^{\mathrm{d}}
$$

Alternatively, one may split force $F_{i j}$ into its tangential and normal components, and isolate, in the latter, the capillary force from the elastic one. This results in a decomposition of stresses into the contribution $\sigma_{\alpha \beta}^{\mathrm{Ne}}$ of normal elastic forces in contacts, the one of tangential contact forces, $\sigma_{\alpha \beta}^{\mathrm{T}}$, and that of capillary forces, $\sigma_{\alpha \beta}^{\text {cap }}$, the latter incorporating both contacts and distant interactions through liquid bridges:

$$
\sigma_{\alpha \beta}=\sigma_{\alpha \beta}^{\mathrm{Ne}}+\sigma_{\alpha \beta}^{\mathrm{T}}+\sigma_{\alpha \beta}^{\mathrm{cap}}
$$

To understand the large values of $\sigma_{12}$ observed at small $P^{*}$, one may use Eq. 11, in which all terms of the sum have the same sign. Distant interactions contribute at most (for small $\left.P^{*}\right) 8 \%$ of the sum. Tangential forces account for about $18 \%$ of the total at $P^{*}=0.436$, decreasing to $10 \%$ for $P^{*}=10$. Thus the essential contribution to shear stress is that of normal contact forces, $\sigma_{12}^{\mathrm{N}, \mathrm{c}}$.

For normal stresses, it is instructive to use decomposition (12). Capillary forces are attractive, and thus contribute negatively to $\sigma_{22}=P$, as shown in Fig. 9. As the contribution of tangential forces is vanishingly small, normal elastic forces have to compensate the effect of $\sigma_{22}^{\text {cap }}$, whence $\frac{\sigma_{22}^{\mathrm{Ne}}}{\sigma_{22}}>3$ for $P^{*}=0.436$. Remarkably, the contribution of capillary forces to shear stress, which is also opposite to that of normal repulsive forces, remains modest: $\frac{\sigma_{12}^{\text {cap }}}{\sigma_{12}}$ evolves from about -0.12 at $P^{*}=0.436$ to -0.03 at $P^{*}=10$.

Following a number of recent micromechanical studies of granular materials (Peyneau and Roux 2008b; Azéma and Radjaï 2014), one may relate stresses $\sigma_{\alpha \beta}^{\mathrm{N}}$ due to normal forces to fabric and force anisotropy parameters, an approach that we do not pursue any further here (more indications are provided by Khamseh et al. (2015)). 
Age of contacts and menisci.

One remarkable feature by which systems with capillary cohesion differ in shear flow from dry granular assemblies is the distribution of contact and interaction ages, expressed in terms of strain intervals. Thus Fig. 10 shows that the same pairs of grains may stay in interaction, joined by a meniscus, over several units of strain, the more often the lower $P^{*}$. Those distribution functions decay exponentially for large values, with a characteristic time growing from $1.1 \dot{\gamma}^{-1}$ for $P^{*}=10$ to $1.7 \dot{\gamma}^{-1}$ for $P^{*}=0.436$ - contrasting with the corresponding decay time for the contact age distribution in a dry cohesionless system $\left(P^{*}=\infty\right)$, which is about $0.12 \dot{\gamma}^{-1}$ for $I=0.1$. Interestingly, contact lifetimes might also exceed a few strain units but are considerably smaller, and, unlike meniscus lifetimes, decrease for increasing $I$ in the investigated range. Whereas pairs of dry grains tend to come into contact if oriented within the compression quadrants of the shear flow, and then separate once in the extension quadrant, grains connected by liquid bonds tend to form clusters that survive tumbling motions in the average shear flow over notable strain intervals. Upon increasing $I$, although contacting pairs separate more easily, they tend to remain joined by menisci. Qualitatively, such a feature might explain the slow $I$ dependence of $\mu^{*}$ and $\Phi$ in strongly cohesive systems, and should be related to the reduced fabric anisotropy at small $P^{*}$, as well as to the influence of meniscus volume or force range $D_{0}$ (Khamseh et al. 2015).

\section{Effective Pressure Approach}

Although the discussion of the different contributions to shear stress $\sigma_{12}$ does not lead to an explanation of the observed large values of $\mu^{*}$, the large tensile contribution of capillary forces to normal stress (Fig. 9) provides a clue. One may write

$$
\sigma_{22}^{\mathrm{cap}}=-\beta \sigma_{22}
$$

with a coefficient $\beta$ ranging, in the quasistatic limit, from about $0.15\left(P^{*}=10\right)$ to 2.1 $\left(P^{*}=0.436\right)$. (If the result for $P^{*}=0.1$ and $I \sim 0.01$ is added $\beta$ then reaches about 7.2). 
Incidentally, the independence of coefficient $\beta$ on inertia parameter $I$ for $I \leq 0.1$ confirms that the rheological effect of liquid bonds is not easily disrupted by collisions in the presence of moderate inertial effects, as noticed from the distribution of their ages in the previous section.

One may invoke the concept of effective pressure to describe the effect of capillary forces on the shear resistance of the material: the attractive forces create larger repulsive elastic reactions in the contact, corresponding to an effective pressure equal to $(1+\beta) \mathcal{P}$. (Note that one ignores here the small contribution of capillary forces to shear stress). One assumes then that the shear behavior of the material is identical to that of a dry material under such effective normal stress $\sigma_{22}^{\text {eff }}$. This approach leads to the following prediction for the $P^{*}$-dependent quasistatic friction coefficient $\mu_{0}^{*}$ :

$$
\mu_{0}^{*}=(1+\beta) \mu_{0}^{\infty}
$$

in which $\mu_{0}^{\infty}$ denotes the quasistatic internal friction coefficient for dry grains, $P^{*}=\infty$.

The performance of the simple effective pressure prediction for the $P^{*}$ dependence of $\mu_{0}^{*}$ is visualized in Fig. 11. Although the global increase of $\mu_{0}^{*}$ is captured, it is overestimated for the smallest $P^{*}$ values $\left(P^{*}=1\right.$ and below). The relative error in the prediction of $\mu_{0}^{*}$, using the exact, measured value of $\beta$, is about $5 \%$ at $P^{*}=10$, increasing to $20 \%$ at $P^{*}=0.436$ (and the value of $\mu_{0}^{*}$ for $P^{*}=0.1$, about 1.62 , from the data for $I \sim 0.01$ is largely overestimated, at 2.7). Thus, the effective stress approach provides a rough estimate for internal friction increase at small $P^{*}$, but becomes inaccurate in the strong cohesion regime. It cannot be exact for various reasons: while the mechanical properties are supposed to be the same once effective stresses are applied to the dry material, the density is different in the dry and the wet case (with $\Phi$ varying between 0.525 and 0.596 as $P^{*}$ grows from 0.436 to infinity); capillary forces also contribute to shear stress, the force network is bound to be different, etc. 


\section{Mohr-Coulomb Model for Critical States}

Coefficient $\beta$ might actually be predicted as follows. From Eq. 10, one may relate (Agnolin and Roux 2007a) the average pressure, $\mathcal{P}=\operatorname{tr} \underline{\underline{\sigma}} / 3$, to the average normal force $\left\langle F^{\mathrm{N}}\right\rangle$ for all interactions, and to the average, $\left\langle F^{\mathrm{N}} h\right\rangle_{d}$, over pairs in distant interaction, of the product of force by distance $h \leq D_{0}$ :

$$
\mathcal{P}=\frac{\Phi z}{\pi a^{2}}\left\langle F^{\mathrm{N}}\right\rangle+\frac{\Phi z_{\mathrm{d}}}{\pi a^{3}}\left\langle F^{\mathrm{N}} h\right\rangle_{d}
$$

As normal stress differences are small, ratio $\frac{\mathcal{P}}{\sigma_{22}}$ only slightly differs from 1 (about 0.95 ) at small $I$. In formula 15 , the second term of the r.h.s. might be neglected, as it contributes less than $2 \%$ of the pressure. Contacts $\left(z_{c}\right.$, on average, per grain) carry capillary force $-F_{0}$, while distant forces $\left(z_{d}\right.$ per grain) average to a fraction of $-F_{0}$. From (15) the capillary contribution to pressure $\mathcal{P}$ is bracketed as $-\frac{\Phi z F_{0}}{\pi a^{2}} \leq \mathcal{P}^{\text {cap }} \leq-\frac{\Phi z_{c} F_{0}}{\pi a^{2}}$, in which $z$ denotes the total coordination number, $z=z_{c}+z_{d}$. Dividing by $\sigma_{22}$, one obtains:

$$
-\frac{\Phi z}{\pi P^{*}} \leq \frac{\mathcal{P}^{\text {cap }}}{\sigma_{22}} \leq-\frac{\Phi z_{c}}{\pi P^{*}}
$$

Ignoring the small difference between $\mathcal{P}^{\text {cap }}$ and $\sigma_{22}^{\text {cap }},(16)$ provides an estimate of coefficient $\beta$ defined in (13). Thus the value of $\beta$ for reduced pressure $P^{*}=0.436$ is predicted between 1.9 and 2.3 (and for $P^{*}=0.1$, it should reach about 8). Relation 16 also suggests that $\beta$ is roughly proportional to $1 / P^{*}$ :

$$
\beta \simeq b / P^{*}, \text { with } \frac{z_{c} \Phi}{\pi} \leq b \leq \frac{z \Phi}{\pi} .
$$

Given the moderate variations of coordination numbers and density with $P^{*}$ in the investigated range, one might choose a constant coefficient $b$ in (17). Eq. 14, on multiplying by $\sigma_{22}$, then takes the form of a Mohr-Coulomb relation:

$$
\sigma_{12}=c+\mu_{1}^{*} \sigma_{22}
$$


This relation, a classical criterion for plastic failure (Wood 1990; Biarez and Hicher 1993; Richefeu et al. 2006; Andreotti et al. 2013), defines a macroscopic cohesion $c$, and an internal friction coefficient $\mu_{1}^{*}$, valid in simple shear for whatever normal stress $\sigma_{22}$. Here, assuming a constant coefficient $b$ in (17), the Mohr-Coulomb relation is predicted to hold with the same value of internal friction as in the dry case, $\mu_{1}^{*}=\mu_{0}^{\infty}$, while macroscopic cohesion $c$ is given by

$$
c=\frac{b \mu_{0}^{\infty} F_{0}}{a^{2}}=\frac{b \pi \mu_{0}^{\infty} \Gamma}{a}
$$

This estimate of the macroscopic cohesion in the Mohr-Coulomb sense is very similar to the one obtained by Richefeu et al. (2006), by a different route. In Fig. 11, the prediction of static friction coefficient $\mu_{0}^{*}$ as a function of $1 / P^{*}$ using $\beta$ as deduced from (17), with coefficient $b$ equal to the middle point of the specified interval, viz. $b=\frac{\left(z_{c}+z\right) \Phi}{2 \pi}$, is shown to perform quite well for $P^{*} \geq 1$, failing at small $P^{*}$, when the effective stress approach with the exact value of $\beta$ fails too.

In general, assuming a Mohr-Coulomb criterion for critical states to apply with a $P^{*}$ independent value of cohesion $c$ implies, upon dividing (18) by $\sigma_{22}=P^{*} F_{0} / a^{2}$, that the quasistatic friction coefficient $\mu_{0}^{*}$ should vary linearly with $1 / P^{*}$ :

$$
\mu_{0}^{*}=\frac{\sigma_{12}}{\sigma_{22}}=\mu_{1}^{*}+\frac{a^{2} c}{F_{0} P^{*}}
$$

The Mohr-Coulomb representation of yield stresses might thus be used as an approximation for $P^{*} \geq 1$, with $a^{2} c / F_{0} \simeq 0.27$, but the observed sublinear increase of $\mu_{0}^{*}$ with $1 / P^{*}$ in Fig. 11 (see the result for $P^{*}=0.436$, and the subplot including value $\mu_{0}^{*} \simeq 1.6$ for $\left.P^{*}=0.1\right)$ clearly precludes the definition of unique values of macroscopic cohesion and friction coefficient according to (18) for smaller pressures.

\section{CONCLUSIONS}

We now provide a quick summary of the main results on both compression and steady shear flow, and end with a discussion, in which perspectives for future work are evoked. 


\section{Isotropic Compression}

Model wet granular assemblies exhibit the same striking differences with cohesionless systems under compression that the simpler, mostly 2D models of the recent literature: stability of loose structures under low $P^{*}$, plastic behaviour in isotropic compression with hardening expressed by the overconsolidation pressure, linear variation of void ratio with $\log P$ in some range - a qualitative behaviour common to many cohesive particulate materials. Comparisons with experimental observations are possible. Although the sensitivity of the results to quite a few model features still needs to be assessed, one may tentatively conclude that the final state, obtained after sufficient overconsolidation, should be independent of the initial configurations and of some aspects of the compression procedure. This state is not affected by the removal of capillary forces, and may be regarded as the result of an ideal, homogeneous and isotropic version of the moist tamping assembling process (Frost and Park 2003; Benahmed et al. 2004). It is looser (see Figs. 2(b) and 5) than directly compressed packs of dry grains, and could qualify as a reference loose state.

\section{Shear Flow and Critical States}

The main rheological influence of capillary adhesion on critical state and shear flow is a strong increase of shear resistance (or apparent friction $\mu^{*}$ ) as $P^{*}$ decreases, even though as $P^{*}$ reaches values of order 0.1 , the strong localization tendency hinders the identification of constitutive laws for homogeneous flow. Meanwhile, density and coordination numbers variations with $P^{*}$ are slower. In the presence of capillary forces, clusters of particles joined by liquid bridges may survive strain intervals of several units, and the compressive role of attractive forces is not as immediately disturbed as the one of the externally applied normal stress upon increasing shear rate and inertial effects. A simple effective stress approach may quantitatively account for the shear resistance trend in good approximation as long as $P^{*} \geq 1$. The Mohr-Coulomb criterion approximately describes critical states in the same reduced pressure range, but is no longer applicable at lower $P^{*}$. Many results (regarding, in particular, normal stress differences, fabric anisotropy, sensitivity to meniscus volume... ) 
are deferred to a more detailed paper submitted by some of the present authors (Khamseh et al. 2015). A major concern, the shear banding instability affecting low $P^{*}$ shear flows, should certainly be addressed in a systematic study as well.

\section{Discussion}

Our simple model of wet grains reveals many new behaviors, compared to dry materials, and provides means for a critical review of macroscopic phenomenological laws (compression curve, Mohr-Coulomb criterion). While some phenomena were already explored in 2D cohesive models, modeling more realistic 3D systems reveals quantitative differences (e.g., a much stronger enhancement of shear resistance), and should permit experimental confrontations (Pierrat et al. 1998; Richefeu et al. 2006). The present paper did not discuss the influence of liquid saturation within the pendular range, and our model with constant meniscus volume does not strictly maintain a fixed water content in the material. Such issues are discussed separately for compression (Than et al. 2015) and shear flow (Khamseh et al. 2015) in forthcoming publications. We could check that a correction of the model in which capillary forces are slightly more accurately described does not significantly change the results. Similarly, a correction of meniscus volume to ensure a constant total liquid volume brings only hardly noticeable changes to compression or shear behavior. Rheological properties vary moderately through the pendular range (Khamseh et al. 2015) (with a very small density change and a variation of about $20 \%$ of $\mu_{0}^{*}$ at $P^{*}=0.436$ ).

A more serious limitation of our model is its inability to deal with saturations exceeding the pendular regime. Numerical models for higher saturation levels, resorting, e.g., to a lattice Boltzmann discretization of the interstitial liquid, are currently being developed (Delenne et al. 2015). Even in the small saturation range, though, the results for compression and shear show that the material behavior is considerably enriched compared to dry granular systems. One obvious, broad perspective is the exploration of the large unknown territory that separates isotropically assembled states from critical states, the simulation of deviatoric loads and the assessment of the applicability of macroscopic models of cohesive soils. It 
would be interesting to explore the effects of resistance to rolling, due to surface asperities, on the material behavior in the presence of capillary cohesion. Even a small rolling resistance was observed to have important effects on the behavior of cohesive systems in two dimensions (Gilabert et al. 2008). It might be viewed as a first step towards the modeling of non-spherical objects (Estrada et al. 2011), but the geometry of liquid bridges joining objects with flat or angular surfaces might entail different force laws, and the effects of capillarity should be investigated in such cases.

23 Than et al., revised version, July 9, 2015 


\section{APPENDIX I. REFERENCES}

Agnolin, I. and Roux, J.-N. (2007a). "Internal states of model isotropic granular packings.

I. Assembling process, geometry, and contact networks." Phys. Rev. E, 76(6), 061302.

Agnolin, I. and Roux, J.-N. (2007b). "Internal states of model isotropic granular packings.

II. Compression and pressure cycles." Phys. Rev. E, 76(6), 061303.

Allen, M. and Tildesley, D. (1987). Computer simulations of liquids. Oxford University Press, Oxford.

Andreotti, B., Forterre, Y., and Pouliquen, O. (2013). Granular Media: Between Fluid and Solid. Cambridge University Press, Cambridge, UK.

Azéma, E. and Radjaï, F. (2014). "Internal struture of inertial granular flows." Phys. Rev. Lett., 112, 078001.

Azéma, E., Radjaï, F., and Dubois, F. (2013). "Packings of irregular polyhedral particles: Strength, structure, and effects of angularity." Phys. Rev. E, 87, 062223.

Azéma, E., Radjaï, F., and Roux, J.-N. (2015). "Internal friction and absence of dilatancy of packings of frictionless polygons." Phys. Rev. E, 91, 010202(R).

Benahmed, N., Canou, J., and Dupla, J.-C. (2004). "Structure initiale et propriétés de liquéfaction statique d'un sable." Comptes-Rendus Académie des Sciences, Mécanique, $332,887-894$.

Biarez, J. and Hicher, P.-Y. (1993). Elementary Mechanics of Soil Behaviour. A. A. Balkema, Rotterdam.

Bruchon, J.-F., Pereira, J.-M., Vandamme, M., Lenoir, N., Delage, P., and Bornert, M. (2013a). "Full 3d investigation and characterisation of capillary collapse of a loose unsaturated sand using x-ray ct.." Granular Matter, 15(6), 783-800.

Bruchon, J.-F., Pereira, J.-M., Vandamme, M., Lenoir, N., Delage, P., and Bornert, M. (2013b). "X-ray microtomography characterisation of the changes in statistical homogeneity of an unsaturated sand during imbibition.." Géotechnique Letters, 3, 84-88.

Cumberland, D. and Crawford, R. (1987). The Packing of Particles. Elsevier, Amsterdam. 
da Cruz, F., Emam, S., Prochnow, M., Roux, J.-N., and Chevoir, F. (2005). "Rheophysics of dense granular materials: discrete simulation of plane shear flows." Phys. Rev. E, 72, 021309.

Delenne, J.-Y., Richefeu, V., and Radjaï, F. (2015). "Liquid clustering and capillary pressure in granular media." J. Fluid Mech., 762, R5.

Donev, A., Torquato, S., and Stillinger, F. H. (2005). "Pair correlation function characteristics of nearly jammed disordered and ordered hard-sphere solids." Phys. Rev. E, 71(1), 011105.

Elata, D. and Berryman, J. G. (1996). "Contact force-displacement laws and the mechanical behavior of random packs of identical spheres." Mechanics of Materials, 24, 229-240.

Estrada, N., Azéma, E., Radjaï, F., and Taboada, A. (2011). "Identification of rolling resistance as a shape parameter in sheared granular media.." Phys. Rev. E, 84, 011306.

Forterre, Y. and Pouliquen, O. (2008). "Flows of dense granular media." Annu. Rev. Fluid Mech., 40, 1-24.

Frost, J. D. and Park, J.-Y. (2003). "A critical assessment of the moist tamping technique." ASTM Geotechnical Testing Journal, GTJ20039850-261.

Gilabert, F. A., Roux, J.-N., and Castellanos, A. (2007). "Computer simulation of model cohesive powders: Influence of assembling procedure and contact laws on low consolidation states." Phys. Rev. E, 75(1), 011303.

Gilabert, F. A., Roux, J.-N., and Castellanos, A. (2008). "Computer simulation of model cohesive powders: Plastic consolidation, structural changes, and elasticity under isotropic loads." Phys. Rev. E, 78(1), 031305.

Hatano, T. (2007). "Power-law friction in closely packed granular materials." Phys. Rev. E, 75, 060301(R).

Herminghaus, S. (2005). "Dynamics of wet granular matter." Advances in Physics, 54, 3, $221-261$.

Khamseh, S., Roux, J.-N., and Chevoir, F. (2015). "Flow of wet granular materials: a 
numerical study.", submitted for publication in Phys. Rev. E.

Kohonen, M. M., Geromichalos, D., Scheel, M., Schier, C., and Herminghaus, S. (2004). "On capillary bridges in wet granular materials." Physica A, 339, 7-15.

Kruyt, N. P. and Rothenburg, L. (2014). "On micromechanical characteristics of the critical state of two-dimensional granular materials." Acta Mechanica, 225(8), 2301 - 2318.

Kudrolli, A. (2008). "Granular matter: Sticky sand." Nature Materials, 7, 174-175.

Lian, G., Thornton, C., and Adams, M. (1993). "A theoretical-study of the liquid bridge forces between 2 rigid spherical bodies." J. Coll. Inter. Sci., 161, 138-147.

Magnanimo, V., La Ragione, L., Jenkins, J. T., Wang, P., and Makse, H. A. (2008). "Characterizing the shear and bulk moduli of an idealized granular material." Europhys. Lett., $81,34006$.

Maugis, D. (1987). "Adherence of elastomers: Fracture mechanics aspects." Journal of Adhesion Science and Technology, 1, 105-134.

Mitarai, N. and Nori, F. (2006). "Wet granular materials." Advances in Physics, 55, 1, 1-45.

Mitchell, J. and Soga, K. (2005). Fundamentals of Soil Behavior. Wiley.

O’Hern, C., Silbert, L. E., Liu, A. J., and Nagel, S. R. (2003). “Jamming at zero temperature and zero applied stress: The epitome of disorder." Phys. Rev. E, 68(1), 011306.

Peyneau, P.-E. and Roux, J.-N. (2008a). "Frictionless bead packs have macroscopic friction, but no dilatancy.." Phys. Rev. E, 78, 011307.

Peyneau, P.-E. and Roux, J.-N. (2008b). "Solidlike behavior and anisotropy in rigid frictionless bead assemblies." Phys. Rev. E, 78, 041307.

Pierrat, P., Agrawal, D. K., and Caram, H. S. (1998). "Effect of moisture on the yield locus of granular materials: theory of shift." Powder Tech., 99, 220-227.

Pitois, O., Moucheront, P., and Chateau, X. (2000). "Liquid bridge between two moving spheres: An experimental study of viscosity effects." J. Coll. Interf. Sci., 231, 26-31.

Radjaï, F., Delenne, J.-Y., Azéma, E., and Roux, S. (2012). "Fabric evolution and accessible geometrical states in granular materials." Granular Matter, 14, 259-264. 
Radjaï, F. and Dubois, F. (2011). Discrete-element modeling of granular materials. Wiley.

Radjaï, F. and Richefeu, V. (2009). "Bond anisotropy and cohesion of wet granular materials." Phil. Trans. R. Soc. A, 367, 5123-5138.

Radjaï, F., Troadec, H., and Roux, S. (2004). "Basic features of granular plasticity." Granular Materials: Fundamentals and Applications, S. J. Antony, W. Hoyle, and Y. Ding, eds., Cambridge, Royal Society of Chemistry, 157-183.

Richefeu, V., El Youssoufi, M., and Radjaï, F. (2006). "Shear strength properties of wet granular materials." Phys. Rev. E, 73, 051304.

Rognon, P., Roux, J.-N., Naaïm, M., and Chevoir, F. (2008). "Dense flows of cohesive granular materials." J. Fluid Mech., 596, 21-47.

Rognon, P., Roux, J.-N., Wolf, D., Naaïm, M., and Chevoir, F. (2006). "Rheophysics of cohesive granular materials." Europhysics Letters, 74, 644-650.

Rothenburg, L. and Kruyt, N. P. (2004). "Critical state and evolution of coordination number in simulated granular materials." International Journal of Solids and Structures, 41(2), $5763-5774$.

Roux, J.-N. and Chevoir, F. (2011). "Dimensional Analysis and Control Parameters." In (Radjaï and Dubois 2011), Chapter 8, 199-232.

Saint-Cyr, B., Szarf, K., Voivret, C., Azéma, E., Richefeu, V., Delenne, J.-Y., Combe, G., Nouguier-Lehon, C., Villard, P., Sornay, P., Chaze, M., and Radjaï, F. (2012). "Particle shape dependence in 2d granular media." EPL, 98(4), 44008.

Scholtès, L., Chareyre, B., Nicot, F., and Darve, F. (2009a). "Micromechanics of granular materials with capillary effects." International Journal of Engineering Science, 47, 64-75.

Scholtès, L., Hicher, P.-Y., Nicot, F., Chareyre, B., and Darve, F. (2009b). "On the capillary stress tensor in wet granular materials." Int. Journal for Numerical and Analytical Methods in Geomechanics, 33, 1289-1313.

Silbert, L. E., Ertaş, D., Grest, G. S., Halsey, T. C., and Levine, D. (2002). "Geometry of frictionless and frictional sphere packings." Phys. Rev. E, 65(3), 031304. 
Soulié, F., Cherblanc, F., El Youssoufi, M., and Saix, C. (2006). "Influence of liquid bridges on the mechanical behaviour of polydisperse granular materials." Int. J. Numer. Anal. Meth. Geomech., 30, 213-228.

Than, V.-D., Roux, J.-N., Tang, A. M., and Pereira, J.-M. (2015). "Numerical simulation of plastic compression of wet granular soils.", to be submitted.

Thornton, C. (2000). "Numerical simulations of deviatoric shear deformation of granular media." Géotechnique, 50, 43-53.

Willett, C., Adams, M., Johnson, S., and Seville, J. (2000). "Capillary bridges between two spherical bodies." Langmuir, 16 (24), 9396-9405.

Wood, D. M. (1990). Soil Behaviour and Critical State Soil Mechanics. Cambridge University Press. 


\section{APPENDIX II. NOTATION}

The following symbols are used in this paper:

$$
\begin{aligned}
& a=\text { spherical grain diameter; } \\
& \alpha=\text { exponent of power law expressing internal friction increase with } I \text {; } \\
& b=\text { proportionality coefficient relating } \beta \text { to } 1 / P^{*} \text {; } \\
& \beta=\text { ratio of capillary contribution to total normal stress in shear flow; } \\
& C=\text { coefficient of } I^{\alpha} \text { for power law increase of } \mu^{*} \text {; } \\
& c=\text { macroscopic cohesion according to Mohr-Coulomb criterion; } \\
& D_{0}=\text { rupture distance of liquid bridge; } \\
& \tilde{E}=\text { Modulus appearing in Hertz law; } \\
& e=\text { void ratio; } \\
& e_{0}=\text { void ratio in critical state; } \\
& e_{\mathrm{iso}}=\text { void ratio in isotropic compression; } \\
& F_{0}=\text { maximum capillary tensile force through liquid bridge; } \\
& \Phi=\text { solid fraction; } \\
& \Phi_{0}=\text { solid fraction in critical state; } \\
& \Phi_{\text {iso }}=\text { solid fraction in isotropic compression; } \\
& \varphi=\text { filling angle in liquid meniscus; } \\
& \Gamma=\text { surface tension of nonsaturating interstitial liquid; } \\
& \dot{\gamma}=\text { shear rate; } \\
& h=\text { distance between particles or contact deflection; } \\
& I=\text { inertial number; } \\
& \kappa=\text { dimensionless contact stiffness number; } \\
& m \text { = grain mass; }
\end{aligned}
$$

29 Than et al., revised version, July 9, 2015 


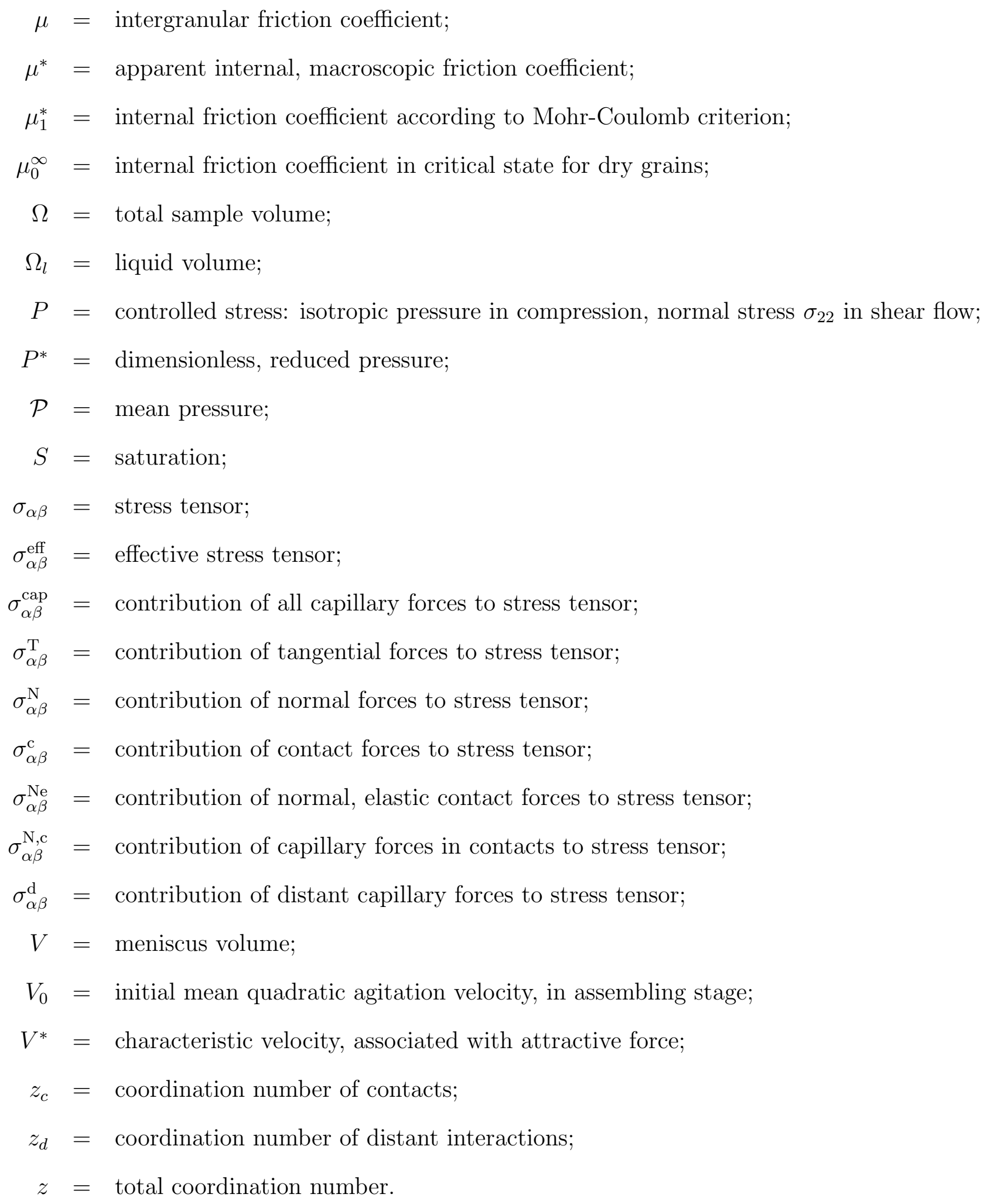




\section{List of Figures}

1 A meniscus between two spherical grains of diameter $a=2 R$, with distance $h$ between solid surfaces, filling angle $\varphi$, contact angle $\theta$. . . . . . . . . . 31

2 (a) Effect of cycle of pressure (in dimensionless form) on void ratio. The straight line fits the curve for intermediate $P^{*}$ values. (b) Same curve, compared to result obtained with dry, cohesionless grains (bottom curve, crosses joined by dark line), with pressure in $\mathrm{kPa}$, for glass beads with $a=0.115 \mathrm{~mm}$, wet by water. . . . . . . . . . . . . . . . . .

3 Effect of different (isotropic) unloading and reloading histories on void ratio. The system does not rearrange along unloading paths $B B^{\prime}, C C^{\prime}, D D^{\prime}, E E^{\prime}$, which are reversible. Path 5 causes plastic response in section $\mathrm{CE}$, along which pressure increases beyond its past maximum. The primary curve (path 4) is then retraced. . . . . . . . . . . . . . . . .

4 Evolution of coordination numbers of contacts $z_{c}$ and of distant interactions $z_{d}$, in the pressure cycle of Fig. ??(a). . . . . . . . . . . . . . . .

$5 \quad$ Void ratio versus reduced pressure $P^{*}$, as cohesive forces are suppressed at the beginning of unloading, starting at different points on the primary compression curve. (In this case the initial state had solid fraction $\Phi_{0}=0.45$ ). . . . . . . 35

6 (a) Internal friction coefficient $\mu^{*}$ and (b) solid fraction $\Phi$ versus $I$ for different values of $P^{*} \ldots \ldots \ldots \ldots \ldots$

7 Main plot: void ratios in primary isotropic compression, $e_{\text {iso }}$ (square dots), and in critical state, $e_{0}$ (crosses with error bars) versus $\log P^{*}$. Inset: detail of variations of solid fraction $\Phi_{\text {iso }}$ in isotropic compression (square dots), and $\Phi_{0}$, in critical state (crosses), for large $P^{*}$ (including the dry case of infinite $\left.P^{*}\right)$, versus $1 / P^{*}$. Dashed lines are drawn to guide the eye. . . . . . . . . 
8 Coordination numbers of contacts $z_{c}$ (left axis, upper curves) and of distant interactions $z_{d}$ (right axis, bottom curves), versus reduced pressure for different values of $I \ldots \ldots \ldots \ldots \ldots$

9 Contribution of capillary forces to normal stress $\sigma_{22}$. . . . . . . . . . . . . . 39

10 Distribution of the age of menisci for different $P^{*}$ values (results for different I indistinguishable on this plot). Inset shows detail of initial decay. . . . . . 40

11 Apparent quasistatic friction coefficient $\mu_{0}^{*}$ versus $1 / P^{*}-$ showing the value of $\mu_{0}^{\infty}$ for $1 / P^{*}=0$. Square dots: numerical results (error bars are smaller); (red) crosses: predictions of (??), with exact coefficient $\beta$; (blue) circles: same with estimated $\beta$. Dotted lines: Mohr-Coulomb models, predicted from (??) (upper line), or fitted to the data in range $P^{*} \geq 1$ (lower straight line). Inset: measured $\mu_{0}^{*}$ versus $1 / P^{*}$, including data point for $P^{*}=0.1$, with MohrCoulomb fit to $P^{*} \geq 1$ data. . . . . . . . . . . . . . . 


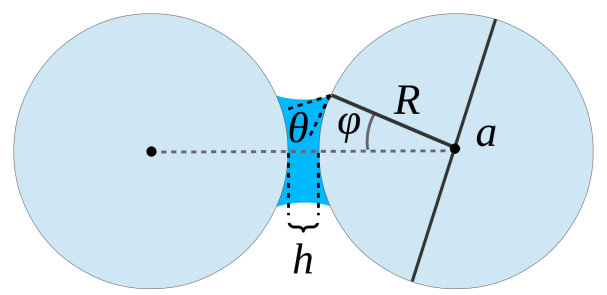

FIG. 1. A meniscus between two spherical grains of diameter $a=2 R$, with distance $h$ between solid surfaces, filling angle $\varphi$, contact angle $\theta$. 


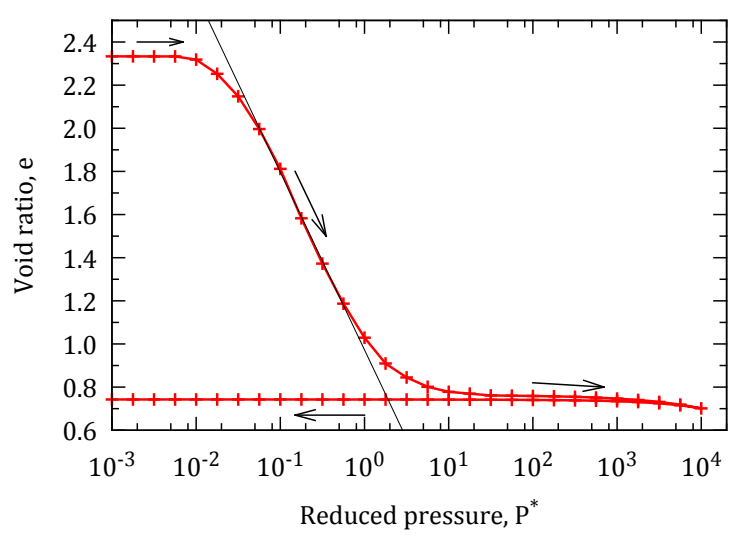

(a)

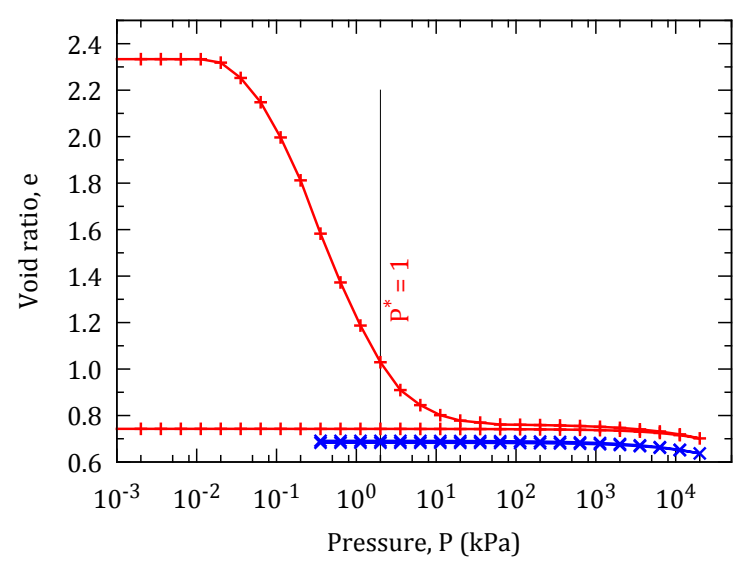

(b)

FIG. 2. (a) Effect of cycle of pressure (in dimensionless form) on void ratio. The straight line fits the curve for intermediate $P^{*}$ values. (b) Same curve, compared to result obtained with dry, cohesionless grains (bottom curve, crosses joined by dark line), with pressure in $\mathbf{k P a}$, for glass beads with $a=0.115 \mathbf{~ m m}$, wet by water. 


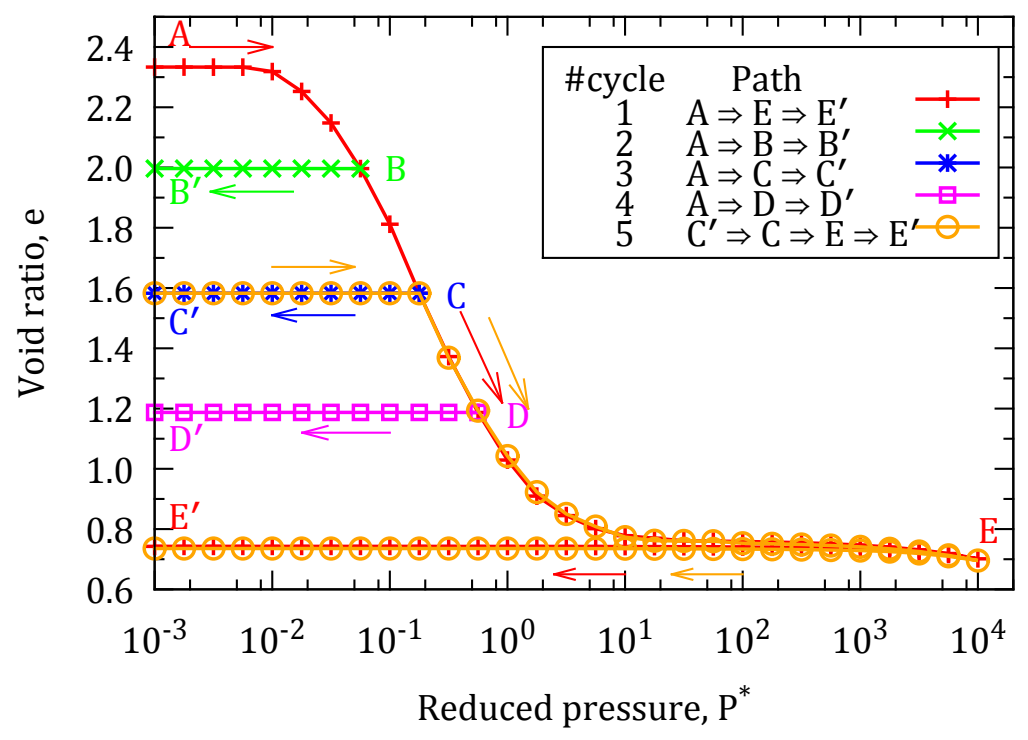

FIG. 3. Effect of different (isotropic) unloading and reloading histories on void ratio. The system does not rearrange along unloading paths $B B^{\prime}, C C^{\prime}, D D^{\prime}, E E^{\prime}$, which are reversible. Path 5 causes plastic response in section $C E$, along which pressure increases beyond its past maximum. The primary curve (path 4 ) is then retraced. 


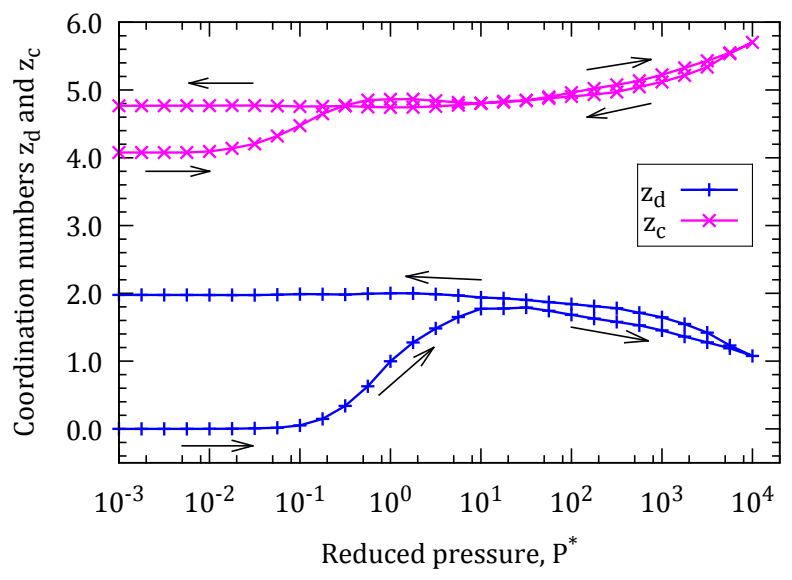

FIG. 4. Evolution of coordination numbers of contacts $z_{c}$ and of distant interactions $z_{d}$, in the pressure cycle of Fig. 2(a). 


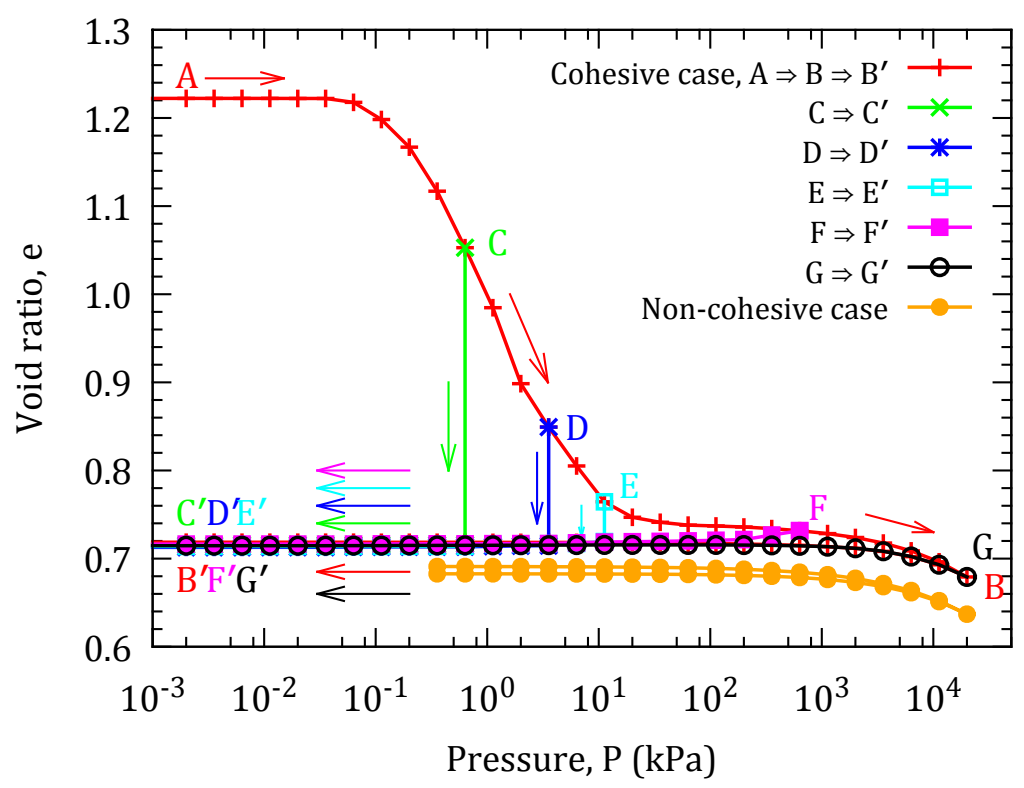

FIG. 5. Void ratio versus reduced pressure $P^{*}$, as cohesive forces are suppressed at the beginning of unloading, starting at different points on the primary compression curve. (In this case the initial state had solid fraction $\Phi_{0}=0.45$ ). 


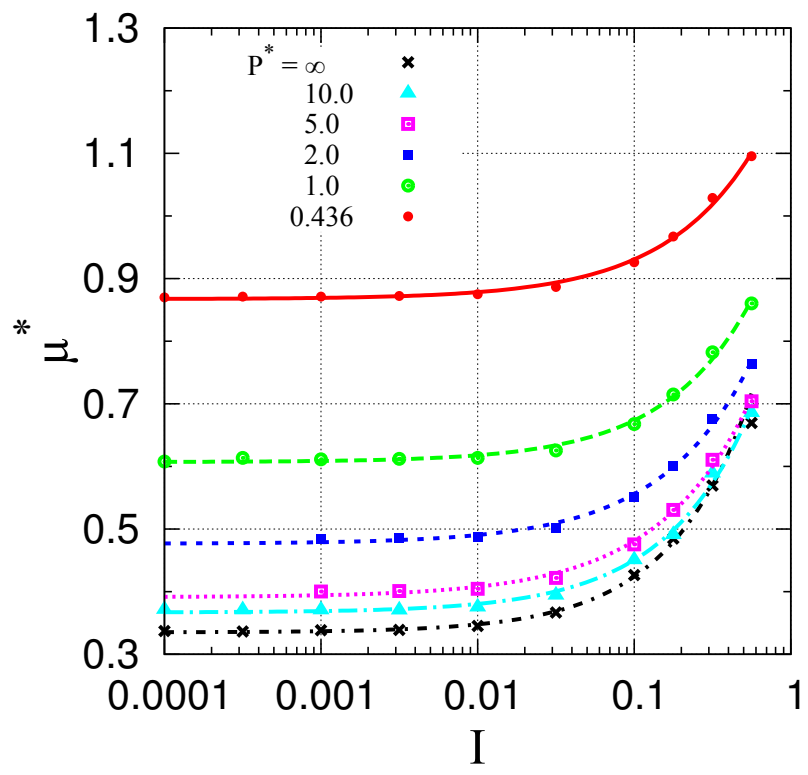

(a)

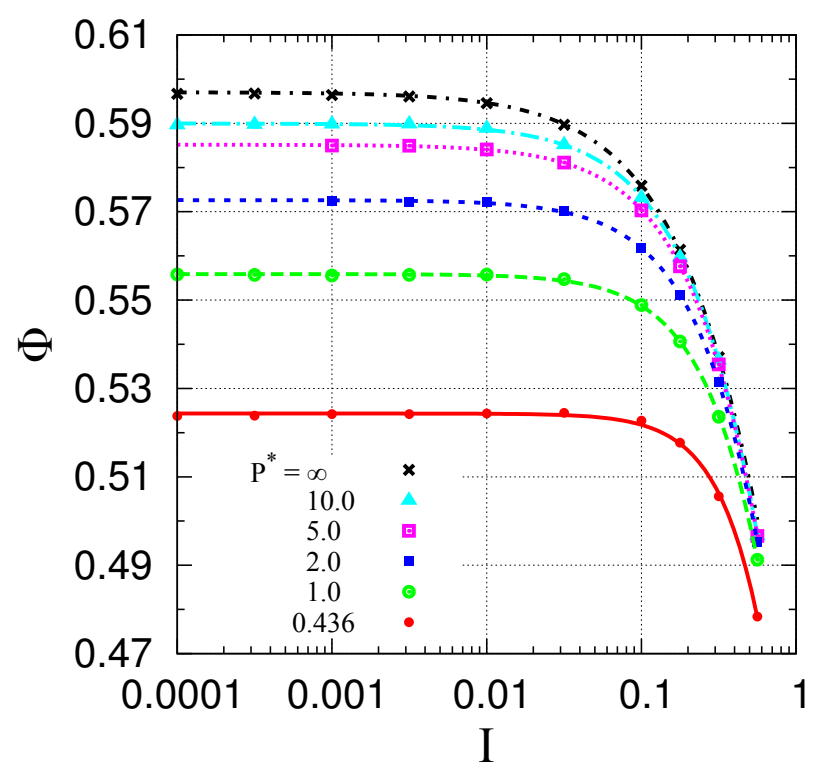

(b)

FIG. 6. (a) Internal friction coefficient $\mu^{*}$ and (b) solid fraction $\Phi$ versus $I$ for different values of $P^{*}$. 


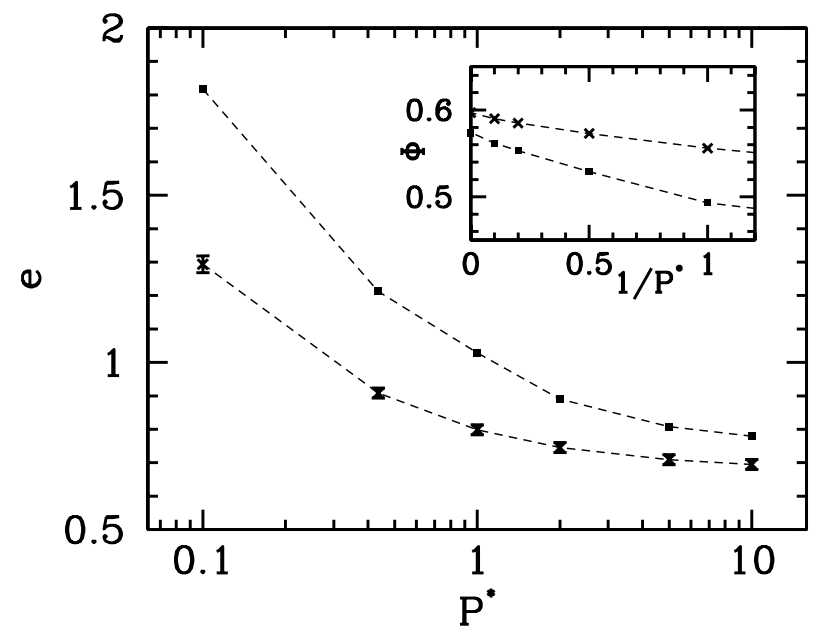

FIG. 7. Main plot: void ratios in primary isotropic compression, $e_{\text {iso }}$ (square dots), and in critical state, $e_{0}$ (crosses with error bars) versus $\log P^{*}$. Inset: detail of variations of solid fraction $\Phi_{\text {iso }}$ in isotropic compression (square dots), and $\Phi_{0}$, in critical state (crosses), for large $P^{*}$ (including the dry case of infinite $P^{*}$ ), versus $1 / P^{*}$. Dashed lines are drawn to guide the eye. 


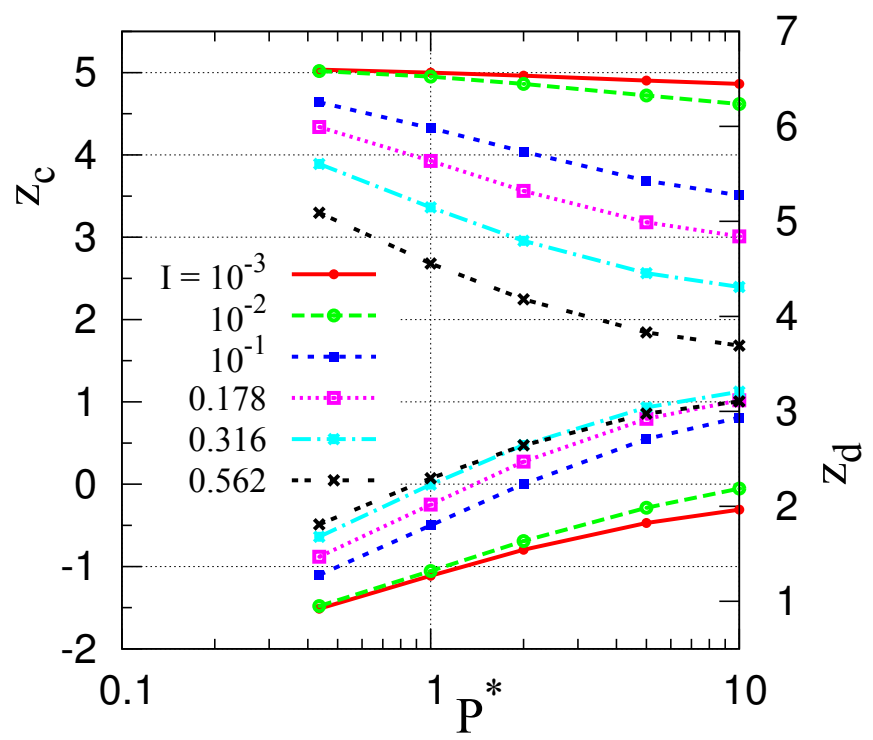

FIG. 8. Coordination numbers of contacts $z_{c}$ (left axis, upper curves) and of distant interactions $z_{d}$ (right axis, bottom curves), versus reduced pressure for different values of $I$. 


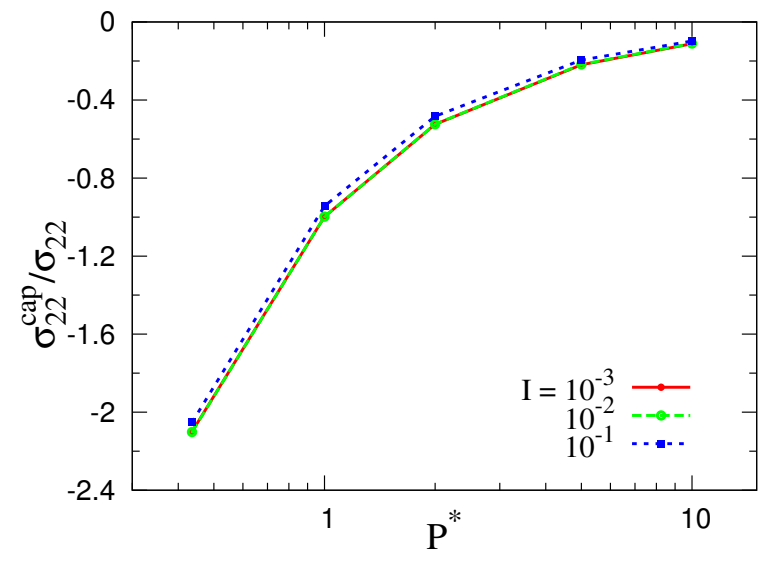

FIG. 9. Contribution of capillary forces to normal stress $\sigma_{22}$. 


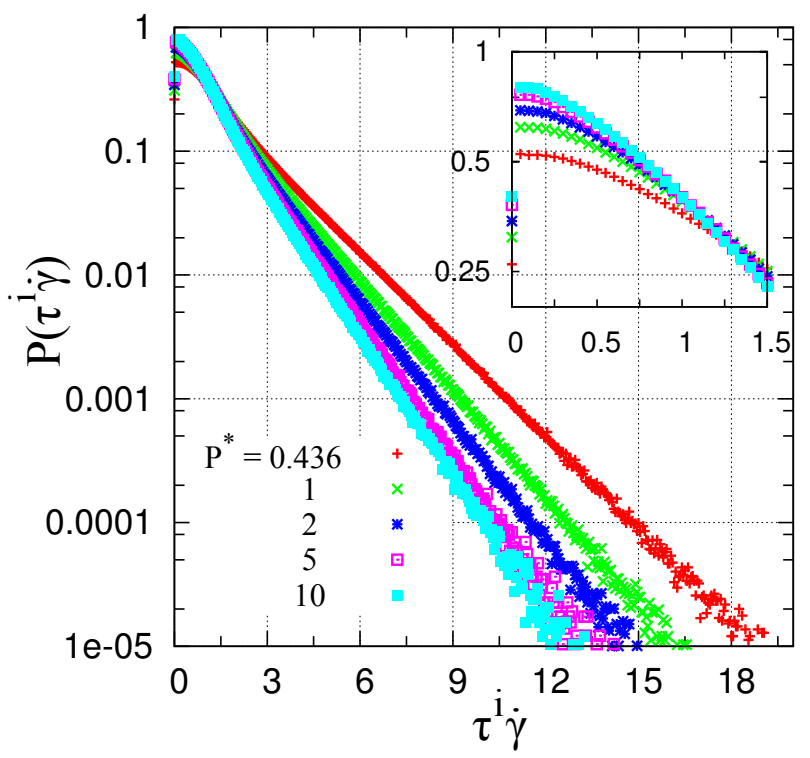

FIG. 10. Distribution of the age of menisci for different $P^{*}$ values (results for different $I$ indistinguishable on this plot). Inset shows detail of initial decay. 


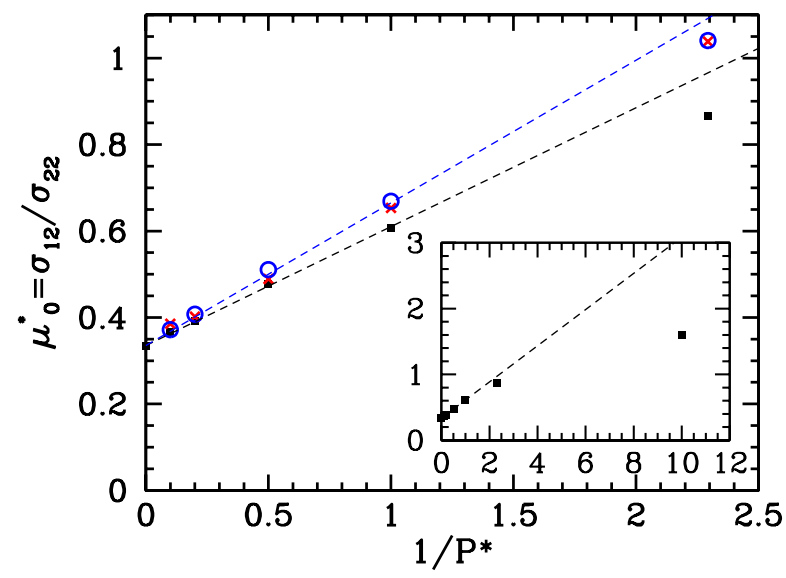

FIG. 11. Apparent quasistatic friction coefficient $\mu_{0}^{*}$ versus $1 / P^{*}$ - showing the value of $\mu_{0}^{\infty}$ for $1 / P^{*}=0$. Square dots: numerical results (error bars are smaller); (red) crosses: predictions of (14), with exact coefficient $\beta$; (blue) circles: same with estimated $\beta$. Dotted lines: Mohr-Coulomb models, predicted from (19) (upper line), or fitted to the data in range $P^{*} \geq 1$ (lower straight line). Inset: measured $\mu_{0}^{*}$ versus $1 / P^{*}$, including data point for $P^{*}=0.1$, with Mohr-Coulomb fit to $P^{*} \geq 1$ data. 